\title{
Searching for antipneumococcal targets: \\ Choline-binding modules as phagocytosis enhancers
}

\author{
Emma Roig-Molina, Manuel Sánchez-Angulo, Jana Seele, \\ Francisco García-Asencio, Roland Nau, Jesús M. Sanz and \\ Beatriz Maestro
}

Number of pages (including this one): $\mathbf{8}$

Number of figures: 4

Number of tables:3 

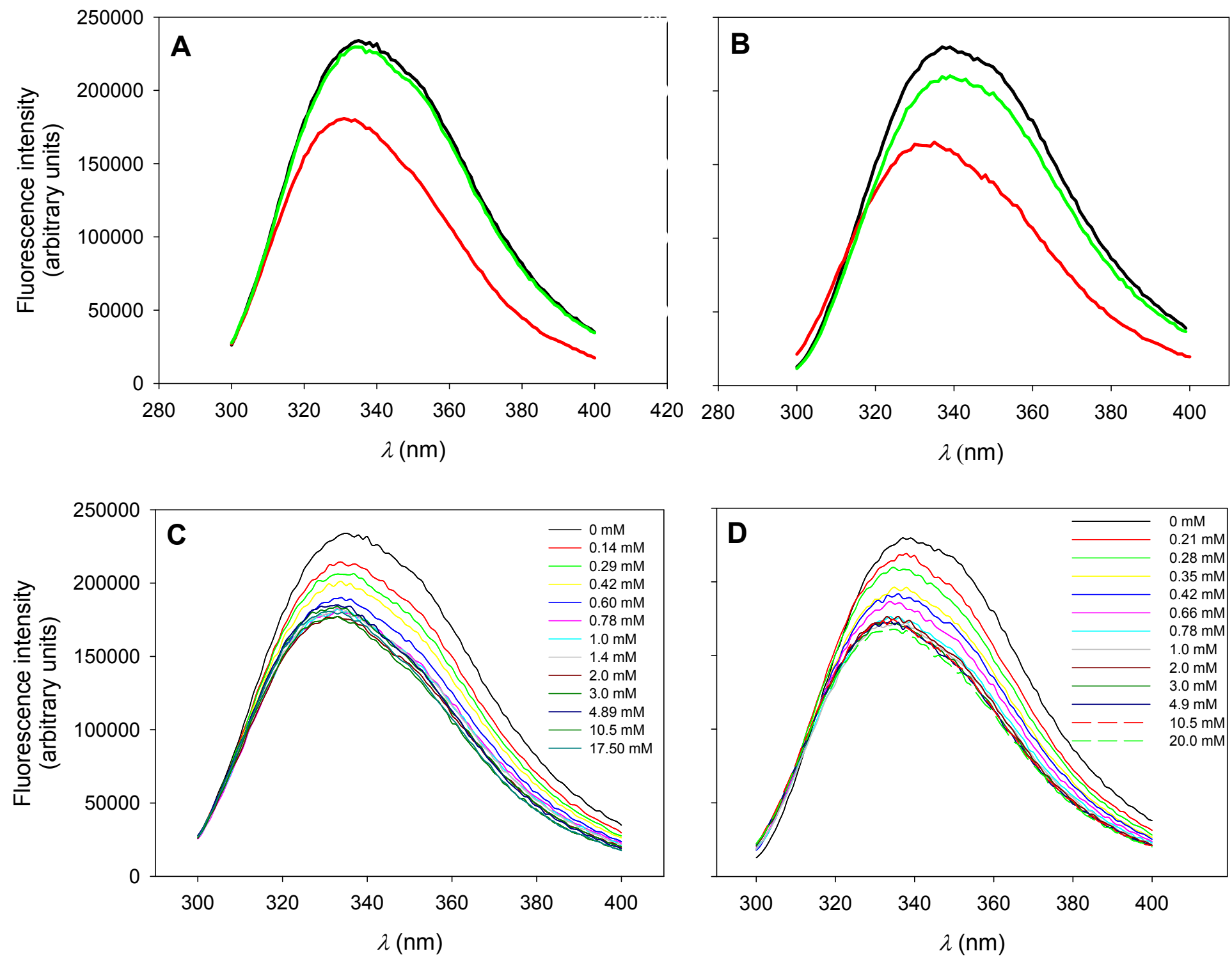

Figure S1. Intrinsic fluorescence emission spectra of C-Cpl1 $(A)$ and C-CbpD $(B)$ in phosphate buffer (black) plus $20 \mathrm{mM} \mathrm{NaCl}$ (green) or $20 \mathrm{mM}$ choline chloride (red); Spectra registered at various choline concentrations for $\mathrm{C}-\mathrm{Cpl} 1(C)$ and $\mathrm{C}-\mathrm{CbpD}(D)$. 


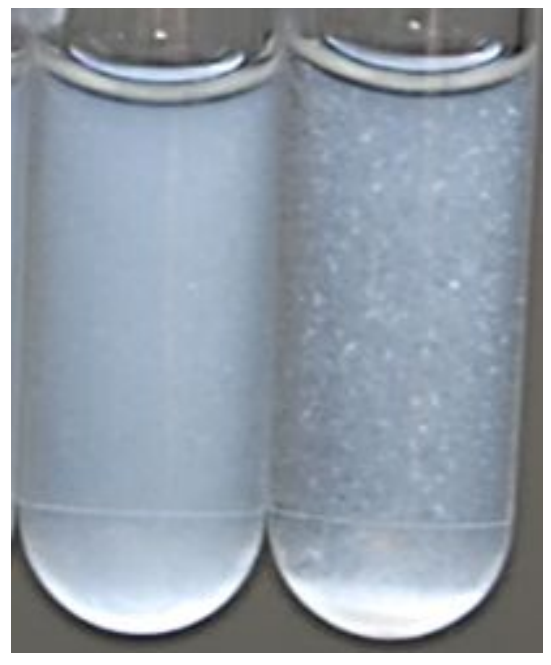

Figure S2. A planktonic culture in the stationary phase of $S$. pneumoniae R6CIB17 in the absence (left) and the presence (right) of C-LytA $1 \mu \mathrm{M}$ 


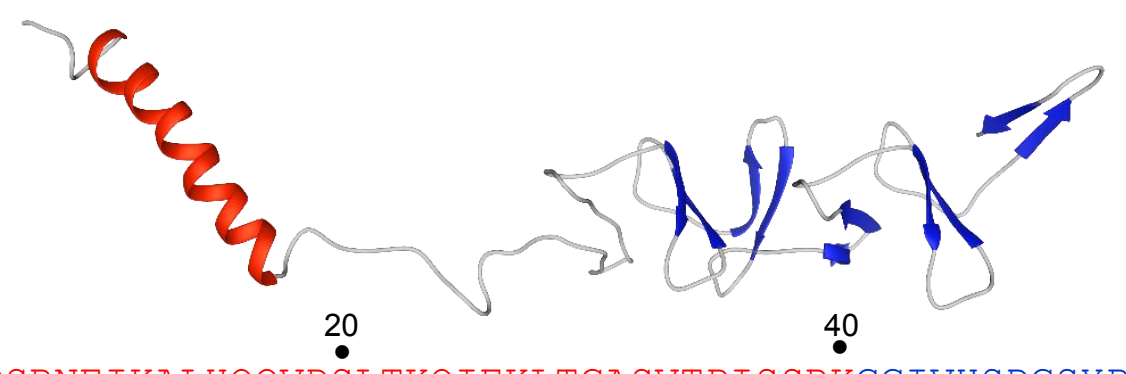

60

MGLGVPSRNEIKALHQQVDSLTKQIEKLTGASVTPISSRKGGIVHSDGSYPKDKFEKING

80

100

120

TWYYFDSSGYMLADRWRKHTDGNWYWFDNSGEMATGWKKIADKWYYFNEEGAMKTGWVKY

140

160

KDTWYYLDAKEGAMVSNAFIQSADGTGWYYLKPDGTLADRPEFTVEPDGLITVK

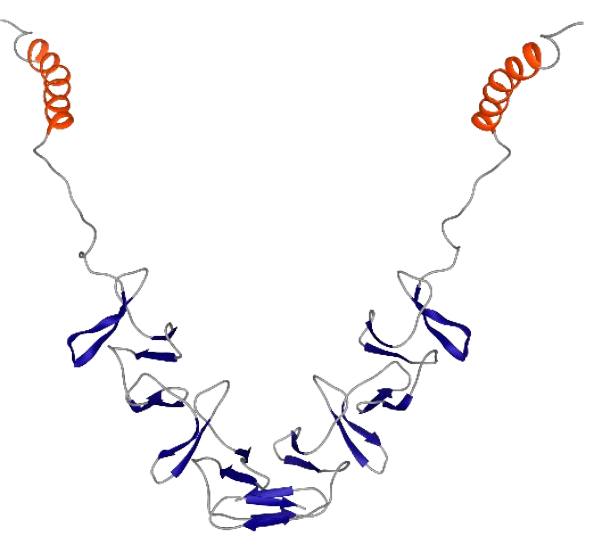

CT-CT

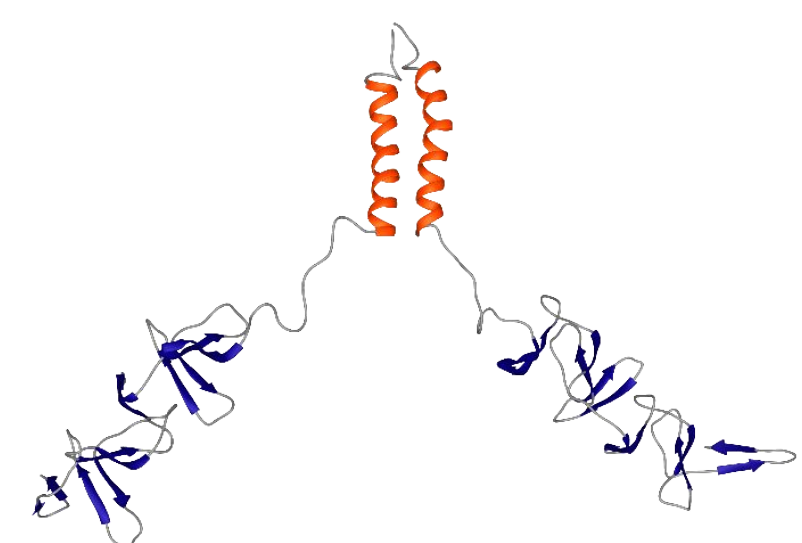

LZ2

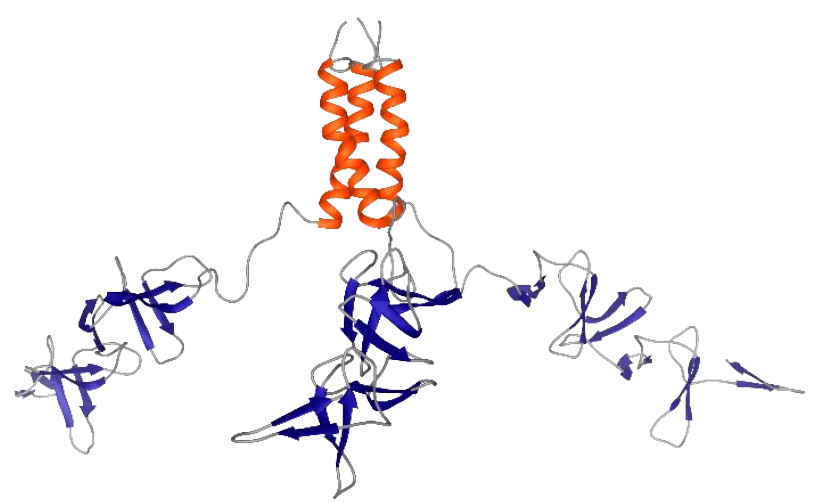

LZ3

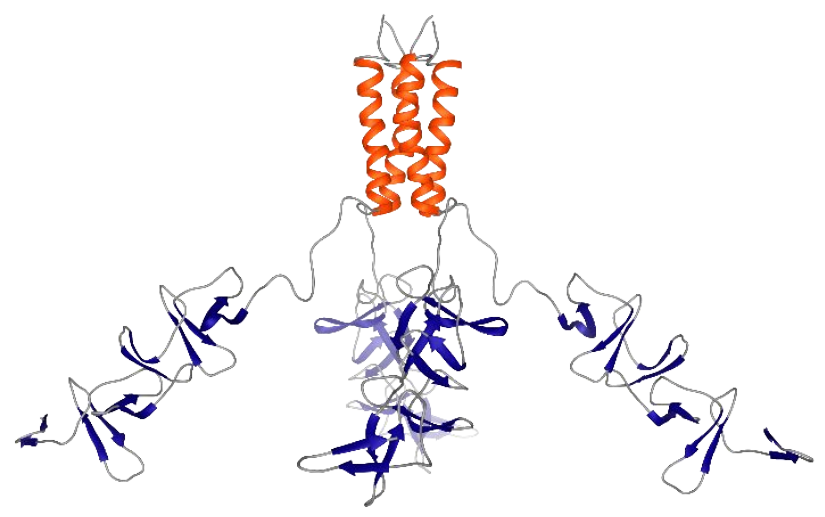

LZ4

Figure S3. Upper panel, amino acid sequence and structural model of the monomer of the LZ-C-LytA protein. Sequence derived from the PhaF phasin is shown in blue, with the predicted leucine-zipper motif underlined. Lower panels: three-dimensional models of LZ-C-LytA oligomers. CT species, dimerization through the Cterminal hairpin of C-LytA; LZ species, oligomerization through the leucine zipper sequence. 


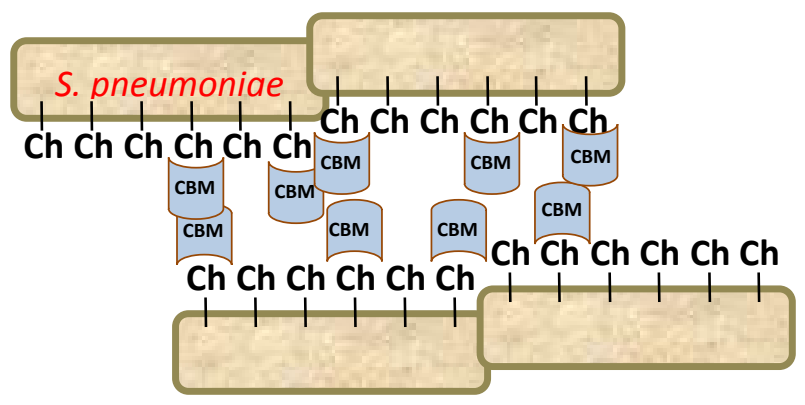

Ch: Choline residue

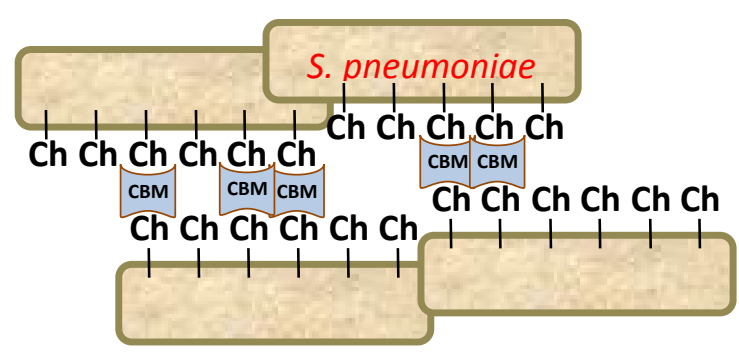

свM Choline-binding module

Figure S4. Possible models for CBM-induced S. pneumoniae agglutination. A, establishment of protein-protein hydrophobic interactions among CBMs adsorbed on the surface of different cells; $B$, simultaneous binding of single CBM molecules to choline residues present in different cells. 


\begin{tabular}{|c|c|c|c|c|c|}
\hline \multirow{2}{*}{ Protein } & \multicolumn{2}{|c|}{$\begin{array}{l}\text { Increment in accessible } \\
\text { surface area }(\triangle A S A)\left(\mathrm{nm}^{2}\right)\end{array}$} & \multicolumn{3}{|c|}{ Estimation of $\Delta C_{p}\left(\mathrm{kcal} \mathrm{mol}^{-1} \mathrm{~K}^{-1}\right)$} \\
\hline & Non polar & Polar & $\begin{array}{l}\text { Pace }^{49} \\
\text { (Eq. 2) }\end{array}$ & $\begin{array}{l}\text { Spolar et } \\
a^{/ 51} \text { (Eq. 3) }\end{array}$ & $\begin{array}{l}\text { Myers et a/50 } \\
\text { (Eq. 4) }\end{array}$ \\
\hline C-Cpl1 & 8025 & 4119 & 2.0 & 2.0 & 1.9 \\
\hline C-CbpD & 5095 & 2558 & 1.2 & 1.3 & 1.2 \\
\hline
\end{tabular}

Table S1. Theoretical estimation of $\Delta C_{p}$ values for C-Cpl1 and C-CbpD proteins. $\triangle A S A$ values were calculated from PDB files with the GetArea utilities. ${ }^{79}$ 


\begin{tabular}{|c|c|c|c|c|}
\hline $\begin{array}{c}\text { Experimental } \\
\text { sedimentation } \\
\text { coefficient (S) }\end{array}$ & $\begin{array}{c}\text { Percentage } \\
\text { of signal } \\
\mathbf{( \% )}\end{array}$ & $\begin{array}{c}\text { Oligomeric state of } \\
\text { model }\end{array}$ & $\begin{array}{c}\text { Oligomerization } \\
\text { mechanism }^{\mathbf{a}}\end{array}$ & $\begin{array}{c}\text { Predicted } \\
\text { sedimentation } \\
\text { coefficient (S) }\end{array}$ \\
\hline 1.09 & 3 & Monomer (19899 Da) & - & 1.60 \\
\hline 2.20 & 27 & Dimer (39781 Da) & CT2 & 2.31 \\
\hline 2.69 & 64 & Trimer (59662 Da) & LZ2 & 2.27 \\
\hline 3.36 & 6 & Tetramer (79543 Da) & LZ4 & 2.81 \\
\hline
\end{tabular}

Table S2. Experimental and predicted sedimentation coefficients of different oligomeric modeled variants of the LZ-C-LytA protein.

a See Figure S4. CT species: dimerization through the C-terminal hairpin of C-LytA; LZ species, oligomerization through the leucine zipper sequence.

$\mathrm{b}$ Estimated using HydroPro utilities (see Materials and Methods) 


\begin{tabular}{|c|c|c|c|}
\hline & \multicolumn{3}{|c|}{ Protein } \\
\cline { 2 - 4 } & C-LytA & C-Cpl1 & LZ-C-LytA \\
\hline $\boldsymbol{V}_{\mathrm{f}}(\boldsymbol{\mu L} / \mathbf{m i n})$ & 20 & 20 & 30 \\
\hline $\boldsymbol{t}_{\text {as }}(\mathbf{s})$ & 250 & 350 & 180 \\
\hline $\boldsymbol{t}_{\text {dis }}(\mathbf{s})$ & 250 & 350 & 150 \\
\hline Regeneration with SDS & No regeneration & $\begin{array}{c}0,5 \% ; 60 \mathrm{~s} \\
(\text { Three times })\end{array}$ & $0,5 \% ; 30 \mathrm{~s}$ \\
\hline
\end{tabular}

Table S3. Conditions of SPR experiments. $V$ f, flow rate; $t_{\mathrm{as}}$, association time; $t_{\mathrm{dis}}$, dissociation time. 